\section{Macrolides, inflammation and the lung microbiome: untangling the web of causality}

\author{
Robert P Dickson, ${ }^{1}$ Alison Morris ${ }^{2,3}$
}

In the past decade, our understanding of the microbiology of the respiratory tract has been revolutionised by the introduction of culture-independent techniques of microbial identification. ${ }^{1}$ The lungs, once considered sterile in the absence of infection, have been consistently shown to contain diverse microbial communities, even in health. ${ }^{2}$ One consistent observation in the field is that in virtually every comparison to date, lung microbiota of healthy subjects differ from the lung microbiota of patients with acute and chronic lung disease. Yet, the field faces a crucial and challenging question: Are bacterial communities in the lung altered because of respiratory disease, or are our patients' lungs diseased because of their altered bacterial communities?

Sorting out cause and effect here is an intimidating task: the microbiome and the host response are so tightly entwined that trying to isolate the effects of each is difficult, both practically and conceptually. Most studies to date in the nascent lung microbiome field have been retrospective and descriptive, replete with provocative correlations, but silent on the direction of causality. Few longitudinal studies have provided the means of determining temporally whether dysbiosis arises before lung disease or vice versa. Given the long chronicity and unpredictability of many lung diseases, prospective cohort studies would need to be impractically large and lengthy to determine temporal precedence. Though animal models hold promise to help unravel mechanisms of pathogenesis ${ }^{34}$ characterising the lung microbiota of small animals is technically challenging, and the relevance of animal-associated microbiota to human respiratory health is far from obvious. Some innovative recent studies

${ }^{1}$ Division of Pulmonary and Critical Care Medicine, University of Michigan Medical School, Ann Arbor, Michigan, USA; ${ }^{2}$ Department of Medicine, University of Pittsburgh, Pittsburgh, Pennsylvania, USA; ${ }^{3}$ Department of Immunology, University of Pittsburgh, Pittsburgh, Pennsylvania, USA

Correspondence to Professor Robert P Dickson, Division of Pulmonary and Critical Care Medicine, University of Michigan Health System, 6220 MSRB III/ SPC 5642, 1150 W. Medical Center Dr., Ann Arbor, MI 48109-5642, USA; rodickso@med.umich.edu have attempted 'lung microbiome transplants' between diseased and non-diseased animals ${ }^{4} 5$ though it has not been established how much of the effect of this exposure is attributable to transferred microbiota and how much is due to transferred host cells, inflammatory mediators and non-bacterial microbiota. ${ }^{6}$ A further challenge is the difficulty of 'isolating' the body's microbial compartments: it is not clear that the microbiota of the lung can be manipulated independently (eg, with antibiotics or via germ-free mice) without also profoundly altering the microbiota of the gut $^{7}$ and, thereby, lung immunity. Despite these difficulties, advancing our understanding of the microbiome's role in lung disease will be crucial if we are ever to target it therapeutically.

But perhaps, ignorant of mechanism, we are already treating the lung microbiome as a therapeutic target. Specifically, macrolide antibiotics (most commonly azithromycin) have recently enjoyed broad and consistent success in the treatment of a wide variety of chronic lung diseases such as cystic fibrosis ${ }^{8}$ bronchiectasis ${ }^{9}$ COPD $^{10}$ bronchiolitis obliterans ${ }^{11}$ and diffuse panbronchiolitis. ${ }^{12}$ Though in recent years the primary rationale for use of macrolides in these diseases has been their pleiotropic immunomodulatory effects, the field has recently confirmed that even in the absence of acute respiratory infection, macrolide therapy alters the lung microbiome. ${ }^{13-15}$ Thus, the unparalleled success of macrolides in the treatment of chronic lung disease presents us with a concrete opportunity to explore the tension outlined above: Do these agents benefit our patients via effects on their microbiota, their host response or both?

In Thorax, Segal et $a l^{14}$ take an ambitious first step towards unravelling this web of cause and effect. They do so by integrating a number of 'next-step' approaches, moving the field beyond the first-pass descriptive accounts that have dominated it to date. The authors studied lung microbiota collected from patients with radiographic emphysema randomised either to azithromycin or placebo. With this study design, they avoided the confounding inherent in prior observational studies and demonstrated that, as predicted, azithromycin does indeed alter lung microbiota. Using the same alveolar specimens, they characterised the cellular and humoral host response, confirming that the inflammatory milieu of the lungs was dampened in patients receiving azithromycin. They then demonstrated that key host-associated and bacteria-associated metabolites were altered following azithromycin exposure, and showed with ex vivo experiments that these metabolitesbut not azithromycin itself-can blunt the alveolar macrophage response to endotoxin challenge. These findings support the hypothesis that the clinical benefit of macrolides and other antibiotics may be attributable as much to their effects on the function of lung bacteria as on their identity and abundance.

As shown in figure 1, the mechanisms behind the benefit of macrolides in chronic lung disease comprise a complex web that spans the host and the microbiome as well as the lungs, the gut and systemic immunity. The current study confirms, consistent with intuition and prior studies, that azithromycin alters the lung microbiome. ${ }^{13-15}$ It also provides proof-of-principle evidence that, via bacterial metabolites, this alteration may modulate the intensity of lung inflammation. It does not preclude, however, any of the numerable alternate routes by which azithromycin may provide benefit. Azithromycin influences systemic inflammation, both directly via its effects on host immune cells ${ }^{16}$ and indirectly via its alteration of gut microbiota. In turn, systemic immunity informs lung immunity, and may directly contribute to disease pathogenesis. ${ }^{17}$ Though certainly plausible that lung microbiota can propel the maladaptive airway inflammation of chronic lung disease, the field has not shown that this reactive inflammation-or the microbiota themselves-directly mediate tissue injury. Finally, as should be expected in any chronic, non-resolving process, a selfreinforcing positive feedback loop is almost certainly in place: tissue injury provokes both lung inflammation and altered lung microbiota via, respectively, damage-associated molecular patterns ${ }^{18}$ and altered environmental growth conditions for lung microbiota. ${ }^{19}$

This mechanistic web is dauntingly complex, and the current study is clearly only an early step towards unravelling it. The authors did not assess for azithromycin's effects on gut microbiota or on systemic immunity, and their ex vivo experiments do not exclude a direct effect of the drug on inflammatory cells other 

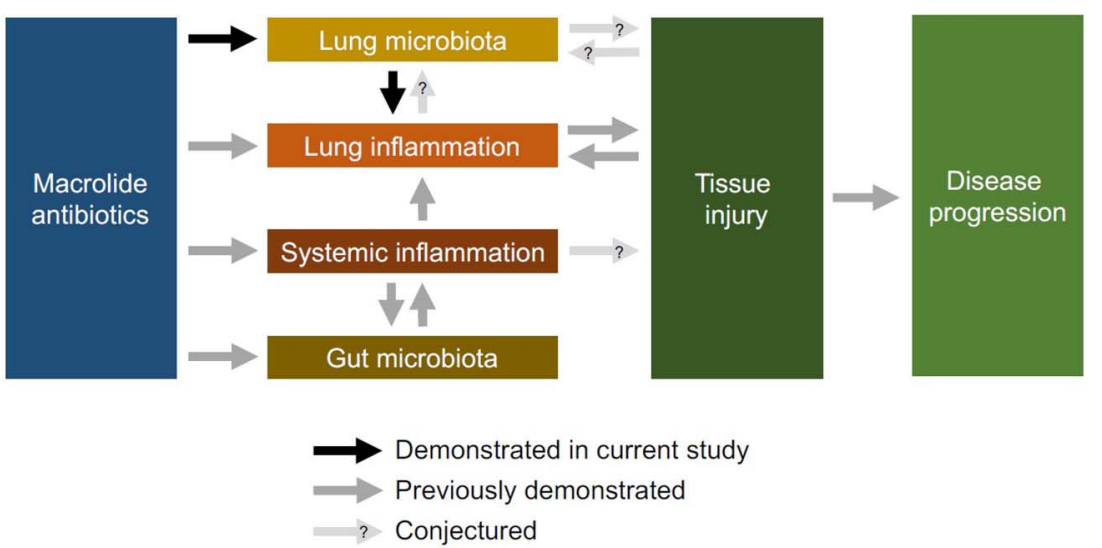

Figure 1 Macrolide antibiotics perturb a complex web of cause and effect in the ecosystem of diseased lungs.

than macrophages (indeed, azithromycin has well-described effects on neutrophils and other key members of the host response ${ }^{16} 20$ ). Their findings also demonstrate a further challenge in metabolomic studies: determining whether a given metabolite in diseased lungs is derived from bacteria or from their host, produced locally in the lung or remotely in the gut, or introduced via diet. Finally, the temporal dynamics of azithromycin's effects on the lung microbiome and its metabolism remains undetermined: the minimum dose, frequency and duration of therapy to achieve clinical and microbiological efficacy have not been established.

Yet more important than this study's limitations is the glimpse it gives us of the future of the field. It is no longer sufficient to merely observe differences in microbiota across disease states or treatment groups: we must leverage these community changes against the host response, the metabolomic activity of host and microbiota, and the controlled observations of in vivo, in vitro and ex vivo experimentation. Microbial ecology of the respiratory tract will no longer merely be a reportable phenomenon unto itself, but instead a powerful tool we can use alongside others to interrogate mechanisms of pathophysiology.

The authors also demonstrate the prudence and power of studying the microbiome as a secondary outcome in randomised controlled trials. Though prior studies have correlated changes in lung microbiota with exposure to common therapies, most studies to date have been observational, highly vulnerable to measured and unmeasured confounders. By characterising the lung microbiome before and after randomised therapies, we will be able to determine both whether the microbiome is altered by treatment and, more importantly, whether it predicts which patients will respond to it: the promise of personalised medicine. We believe that the microbiome of both the lung and the gut should be considered a key secondary outcome in trials of interventions that may be exerting their effects via the microbiome, including antibiotics, immunomodulatory agents, nutritional supplements and even interventions such as weight loss and physical activity. Investigations of microbes other than bacteria will also be important in future studies as we are just beginning to understand the complex web of interactions between bacteria, fungi and viruses. A significant barrier to implementing microbiome analyses in clinical trials is the need for bronchoscopy, and the field would benefit greatly by determining the potential utility of surrogate markers of bacteria and their products. Finally, although the authors did not evaluate changes in pulmonary function or subsequent clinical course in this trial, linking alteration of the microbiome to important clinical outcomes such as pulmonary function, exacerbation frequency or mortality is clearly the holy grail of the field.

Although the interplay of host, microbiota and lung disease weaves a tangled web, we are beginning to unravel its many threads. The study by Segal et al is a first step in illuminating the complex network of effects of a microbiome-altering therapy for lung disease.

\section{Competing interests None declared.}

Provenance and peer review Commissioned; externally peer reviewed.

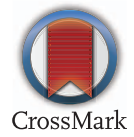

To cite Dickson RP, Morris A. Thorax 2017;72:10-12.
Published Online First 31 October 2016

\section{(P) Linked}

http://dx.doi.org/10.1136/thoraxjnl-2016-208599

Thorax 2017:72:10-12.

doi:10.1136/thoraxjnl-2016-209180

\section{REFERENCES}

1 Dickson RP, Erb-Downward JR, Martinez FJ, et al. The microbiome and the respiratory tract. Annu Rev Physiol 2016;78:481-504.

2 Morris A, Beck JM, Schloss PD, et al. Comparison of the respiratory microbiome in healthy nonsmokers and smokers. Am J Respir Crit Care Med 2013;187:1067-75.

3 Dickson RP, Singer BH, Newstead MW, et al. Enrichment of the lung microbiome with gut bacteria in sepsis and the acute respiratory distress syndrome. Nat Microbiol 2016;1:16113.

4 Poroyko V, Meng F, Meliton A, et al. Alterations of lung microbiota in a mouse model of LPS-induced lung injury. Am J Physiol Lung Cell Mol Physiol 2015:309:L76-83.

5 Yadava K, Pattaroni C, Sichelstiel AK, et al. Microbiota promotes chronic pulmonary inflammation by enhancing il-17a and autoantibodies. Am J Respir Crit Care Med 2016;193:975-87.

6 Bojanova DP, Bordenstein SR. Fecal transplants: What is being transferred? PLOS Biol 2016;14: e1002503.

7 Dickson RP, Cox MJ. The premature invocation of a 'gut-lung axis' may obscure the direct effects of respiratory microbiota on pneumonia susceptibility. Gut 2016; Published Online First: 1 April 2016.

8 Saiman L, Marshall BC, Mayer-Hamblett N, et al. Azithromycin in patients with cystic fibrosis chronically infected with Pseudomonas aeruginosa: a randomized controlled trial. JAMA 2003;290: 1749-56.

9 Wong C, Jayaram L, Karalus N, et al. Azithromycin for prevention of exacerbations in non-cystic fibrosis bronchiectasis (EMBRACE): a randomised, double-blind, placebo-controlled trial. Lancet 2012;380:660-7.

10 Albert RK, Connett J, Bailey WC, et al. Azithromycin for prevention of exacerbations of COPD. N Engl J Med 2011;365:689-98.

11 Yates B, Murphy DM, Forrest IA, et al. Azithromycin reverses airflow obstruction in established bronchiolitis obliterans syndrome. Am J Respir Crit Care Med 2005; 172:772-5.

12 Kudoh S, Azuma A, Yamamoto M, et al. Improvement of survival in patients with diffuse panbronchiolitis treated with low-dose erythromycin. Am J Respir Crit Care Med 1998;157(Pt 1): 1829-32.

13 Slater M, Rivett DW, Williams L, et al. The impact of azithromycin therapy on the airway microbiota in asthma. Thorax 2014;69:673-4.

14 Segal LN, Clemente JC, Wu BG, et al. Randomized, double-blind, placebo-controlled trial with azithromycin selects for anti-inflammatory microbial metabolites in the emphysematous lung. Thorax 2017;72:13-22.

15 Rogers GB, Bruce KD, Martin ML, et al. The effect of long-term macrolide treatment on respiratory microbiota composition in non-cystic fibrosis bronchiectasis: an analysis from the randomised, double-blind, placebo-controlled BLESS trial. Lancet Respir Med 2014;2: 988-96. 


\section{Editorial}

16 Kanoh S, Rubin BK. Mechanisms of action and clinical application of macrolides as immunomodulatory medications. Clin Microbiol Rev 2010;23:590-615.

17 Agustí A, Edwards LD, Rennard SI, et al., Evaluation of COPD Longitudinally to Identify Predictive Surrogate Endpoints (ECLIPSE) Investigators.
Persistent systemic inflammation is associated with poor clinical outcomes in COPD: a novel phenotype. PLOS ONE 2012;7:e37483.

18 Pouwels SD, Heijink IH, ten Hacken NH, et al. DAMPs activating innate and adaptive immune responses in COPD. Mucosal Immunol 2014;7:215-26.
19 Dickson RP, Martinez FJ, Huffnagle GB. The role of the microbiome in exacerbations of chronic lung diseases. Lancet 2014;384:691-702.

20 Culic O, Erakovic V, Cepelak I, et al. Azithromycin modulates neutrophil function and circulating inflammatory mediators in healthy human subjects. Eur J Pharmacol 2002;450:277-89. 